\title{
Downregulation of ZNF278 arrests the cell cycle and decreases the proliferation of colorectal cancer cells via inhibition of the ERK/MAPK pathway
}

\author{
XIAO-QING TIAN* , FANG-FANG GUO* , DAN-FENG SUN, YING-CHAO WANG, LI YANG, \\ SHENG-LIANG CHEN, JIE HONG and JING-YUAN FANG \begin{abstract}
Ministry of Health, State Key Laboratory for Oncogenes and Related Genes, Renji Hospital, School of Medicine, Shanghai Jiao Tong University, Shanghai Institute of Digestive Disease,
\end{abstract} \\ Division of Gastroenterology and Hepatology, Key Laboratory of Gastroenterology and Hepatology, \\ Shanghai 200001, P.R. China
}

Received March 15, 2017; Accepted September 19, 2017

DOI: 10.3892/or.2017.6031

\begin{abstract}
Zinc finger protein 278 is a zinc finger transcription factor encoded on the 22q12.2 chromosome. Previous studies revealed that ZNF278 expression was significantly upregulated in colorectal cancer (CRC) tissue compared to adjacent non-tumor tissue. However, the expression and specific roles of ZNF278 in CRC remain unknown. ZNF278 expression was knocked down using specific siRNAs, which was confirmed by western blotting, and the effects of ZNF278 siRNAs on CRC cell proliferation were investigated. In addition, the effects of ZNF278 overexpression were confirmed by western blotting and cell proliferation assay. Correlations between ZNF278 and the ERK/MAPK pathway were also detected by western blotting. We found that ZNF278 knockdown significantly induced cell cycle arrest, resulting in cyclin D1/E1 downregulation and p21 upregulation. Moreover, we demonstrated that downregulation of ZNF278 decreased the proliferation of CRC cells via inhibition of the extracellular signal-regulated kinase/mitogen-activated protein kinase (ERK/MAPK) pathway for the first time. In conclusion, ZNF278 played a prominent role in the pathogenesis of CRC, and promoted CRC cell proliferation via the ERK/MAPK pathway, suggesting that it may act as a potential target in the diagnosis or treatment of CRC.
\end{abstract}

Correspondence to: Dr Jing-Yuan Fang or Jie Hong, Renji Hospital, School of Medicine, Shanghai Jiao Tong University, 145 Middle Shandong Road, Shanghai 200001, P.R. China

E-mail: fangjingyuan_new@163.com

E-mail: jiehong97@163.com

*Contributed equally

Key words: zinc finger protein 278, ERK-MAPK pathway, cell cycle, cell proliferation, colorectal cancer

\section{Introduction}

Colorectal cancer (CRC) is the third most commonly diagnosed cancer in males and the second in females worldwide (1). The incidence rates of CRC are rapidly increasing in several areas including Eastern European countries and most parts of Asia (2,3). However, the biological and molecular mechanisms underlying CRC development remain largely unclear. Zinc finger protein 278 (ZNF278), also named POZ/BTB and AT-hook-containing zinc finger protein (PATZ), is a transcription factor with seven C2H2-type zinc fingers (4). ZNF278 belongs to the Krueppel $\mathrm{C} 2 \mathrm{H} 2$-type zinc finger protein family. It is a novel zinc finger protein, which is ubiquitously distributed in human tissues. Although the physiological role of ZNF278 is not clear, some experimental evidence suggests that it is a potential transcription suppressor $(4,5)$. Rearrangement of ZNF278 was involved in small round cell sarcoma (6). Indeed, our previous study revealed that ZNF278 expression was increased in $53 \%$ of CRC tissues compared to corresponding non-cancerous tissues. The functional study revealed that ZNF278 promoted cell growth and its knockdown suppressed cell proliferation, indicating that ZNF278 could be a potential proto-oncogene in CRC (7). However, the molecular mechanism of ZNF278 in CRC remains unclear. In the present study, we analyzed the expression of cyclin D1 and E1 by ZNF278-siRNA transfection. Knockdown of ZNF278 induced cell cycle arrest in CRC cell lines. The present study revealed that depletion of ZNF278 may decrease the proliferation of CRC cells via inhibition of the ERK/MAPK pathway.

\section{Materials and methods}

Cell culture and PD98059 treatment. The human CRC cell line HT29 was maintained in McCoy's 5A medium and the CRC cell line SW1116 in RPMI-1640 medium (both from Gibco, Gaithersburg, MD, USA) supplemented with $10 \%$ fetal bovine serum at $37^{\circ} \mathrm{C}$ in a $5 \% \mathrm{CO}_{2}$ incubator. For treatment with PD98059 (Cell Signaling Technology, Danvers, MA, 
USA), the cells were incubated with $20 \mu \mathrm{M}$ PD98059 for $48 \mathrm{~h}$ before harvesting for assessments.

Real-time PCR for four different ZNF278 variants. Total RNAwasisolatedusingTRIzol reagent(Invitrogen/Gibco-BRL, Carlsbad, CA, USA). Total RNA $(1 \mu \mathrm{g})$ was reverse transcribed using the PrimeScript RT reagent kit (Perfect Real-Time; Takara, Tokyo, Japan) to detect relative mRNAs. Relative quantitative data were obtained using the comparative $\mathrm{Ct}$ method on an Applied Biosystem 7900 quantitative PCR system (Applied Biosystems, Foster City, CA) according to the manufacturer's protocol. The specific primers for ZNF278 variants were as follows: variant 1 (GenBank accession no. NM_032050) F, 5'-GCAGTATCTGTAACCGAGAA GGC-3' and R, 5'-ATTTCCCTTCAGGCCCCATG-3'; variant 2 (NM_032051) F, 5'-GAGGGTTGACAGTGGAA GGG-3' and R, 5'-ATTTGGGGGCTCTGACATGG-3'; variant 3 (NM_032052) F, 5'-GCAGTATCTGTAACCGAGG TCTC-3' and R, 5'-CGGACATGCACCTTCTGGAT-3'; variant 4 (NM_014323) F, 5'-AAAACCCACCACGGTGT TCC-3' and R, 5'-CATTTCCCTTCAGGCCCCAT-3'. The Ct values obtained from different samples were compared using the $2^{-\Delta \Delta C t}$ method. GAPDH served as an internal reference gene and the results were presented as the ratio of copies of target genes to GAPDH.

Construction of expression vectors and transfection. To construct the wild-type ZNF278 (GenBank accession no. NM_032050) expression vector, a PCR-generated full length ZNF278 cDNA was inserted into the EcoRI-HindIII sites of the expression vector CMV-MCS-3FLAGSV40-Neomycin. Nested PCR was carried out to amplify the full-length ZNF278 cDNA. The following primers were used: F1, 5'-CGGCGCACCTGCGAGACTACAGA-3' and R1, 5'-TC CCAGCAGTCCCCAGATGGTTGT-3' for the first PCR; and F2, 5'-CCCAAGCTTCCATGGAGCGGGTGAAC-3' and R2, 5'-CCGGAATTCTTTCCCTTCAGGCCCCAT-3' for the second PCR. Before transfection, 5x10 $0^{5}$ HT29 and SW1116 cells were seeded in $6 \mathrm{~cm}$ wells. The cells were transfected with $2 \mu \mathrm{g}$ of either ZNF278 or control plasmid, using FuGENE HD transfection reagent (Promega, Madison, WI, USA), in accordance with the manufacturer's instructions. The cells were collected for measurements $48 \mathrm{~h}$ after transfection.

RNA interference and transient transfections. All transfections were performed using DharmaconFECT (Thermo Fisher Scientific, Inc., Waltham, MA, USA), according to the manufacturer's instructions. ZNF278 small interfering RNA (siRNA) (sense, 5'-GCGCCGAUAUAAUGCUCUUTT-3' and antisense, 5'-AAGAGCAUUAUAUCGGCGCGG-3'); and negative control siRNA (sense, 5'-UUCUCCGAAC GUGUCACGUTT-3' and antisense, 5'-ACGUGACACGUUC GGAGAATT-3') were designed and synthesized by GenePharma (Shanghai, China). ERK2 siRNA was sense, 5'-CACUUGUCAAGAAGCGUUAdTdT-3' and antisense, 5'-CACUUGUCAAGAAGCGUUAdTdT-3'. Non-specific siRNAs (GenePharma) were used as the negative controls. The siRNA was complexed with the transfection reagent in a serum- and antibiotic-free medium for $6 \mathrm{~h}$. After applying the transfection reagents, the cellular medium was replaced with the serum-containing maintenance medium, and the cells were incubated for $48 \mathrm{~h}$. Gene expression silencing was confirmed by western blotting analysis.

Western blot analysis. Western blotting was performed according to the standard protocols, as previously described (8). The primary antibodies used in the present study were as follows: anti-ZNF278 (1:1,000; ab126903; Abcam, Cambridge, UK); anti-p-MEK1/2 (1:2,000; CST9121s); anti-p-ERK1/2 (CST4370s), anti-ERK1/2 (CST9102s), anti-MEK1/2 (CST9122s), anti-cyclin E1 (CST4129s), anti-cyclin D1 (CST2978s) and anti-p21 (CST2947T) (1:1,000) (all from Cell Signaling Technology). Glyceraldehyde-3-phosphate dehydrogenase (GAPDH; KC-5G5; KangChen, Shanghai, China) was used as a loading control. The blots were analyzed using ImageJ 1.43 software and protein expression was normalized to GAPDH and relative to the control.

Cell viability assay. Cell viability was assessed by a tetrazolium salt (WST-8)-based colorimetric assay called Cell Counting Kit-8 (CCK-8; Dojindo, Kumamoto, Japan). Briefly, $5 \times 10^{3}$ cells were transfected with ERK2 siRNA or negative control siRNA after $24 \mathrm{~h}$ of culture in 96 -well plates. At the specified time-points, $10 \mu 1$ of CCK-8 solution was added to each well and the cells were incubated for $1 \mathrm{~h}$. Then, cell viability was determined by measuring the absorbance values at $450 \mathrm{~nm}$ using a microplate reader. Data are expressed as the percentage of viable cells, calculated as: relative viability $(\%)=[\mathrm{A} 450$ (treated) $-\mathrm{A} 450$ (blank)]/[A450 (control) - A450 (blank)] x 100\%.

Statistical analysis. Data are expressed as the mean \pm SEM. Differences between two groups were compared using the Student's t-test. All experiments were repeated at least three times. P-values $<0.05$ were considered statistically significant.

\section{Results}

Effect of ZNF278 siRNA and plasmid transfection on the cell cycle in CRC cells. To confirm which variants of ZNF278 were decreased after ZNF278-siRNA transfection, four pairs of specific primers were designed and synthesized. It was identified that all four ZNF278 variants were downregulated by ZNF278-siRNA transfection (Fig. 1A and B). As a previous study revealed overexpression of ZNF278 in the cells transfected with the pcDNA3.1-ZNF278 plasmid significantly increased the percentage of the $\mathrm{S}$ phase cells and decreased the percentage of the G0/G1 phase cells. Knockdown of ZNF278 expression significantly blocked the cell cycle at the G0/G1 phase (7). The cell cycle analysis revealed that G1 phase arrest was detected in SW1116 cells after depletion of ZNF278, suggesting that knockdown of ZNF278 induced cell cycle arrest. Furthermore, we examined the protein expression of cyclin E1 and D1 and p21, the three key genes involved in cell cycle progression $(9,10)$. Expression of cyclin E1 and cyclin D1 were significantly decreased and p21 was increased after transfection of ZNF278 siRNA compared with the control siRNA in HT29 and SW1116 cells (Fig. 1C and D). Conversely, upregulation of cyclin E1 and D1 and downregultion of p21 were detected by ZNF278 plasmid transfection (Fig. 1E and F). The 
A

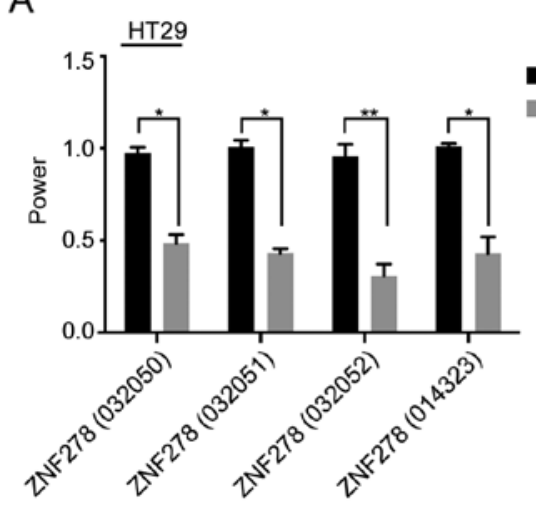

C

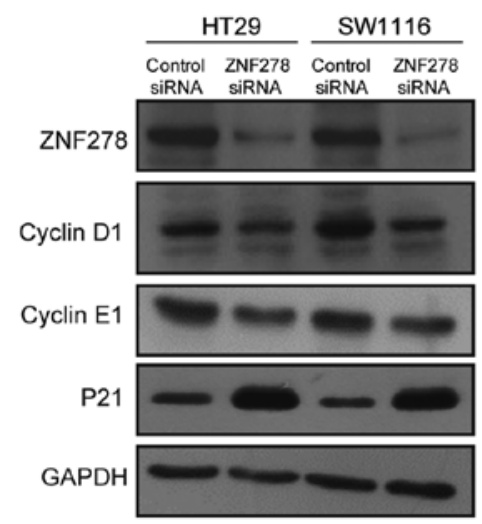

E

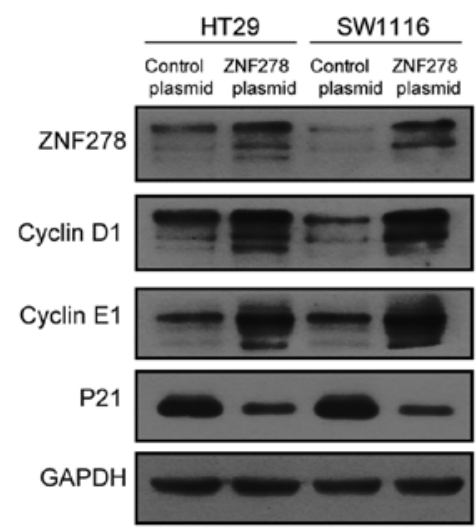

B

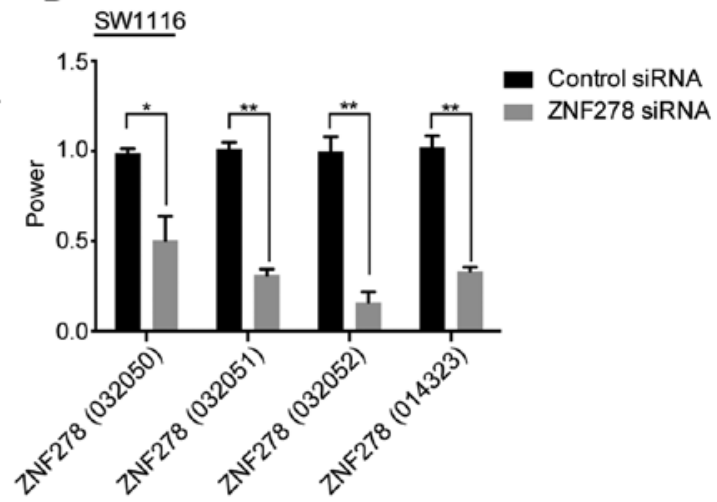

D

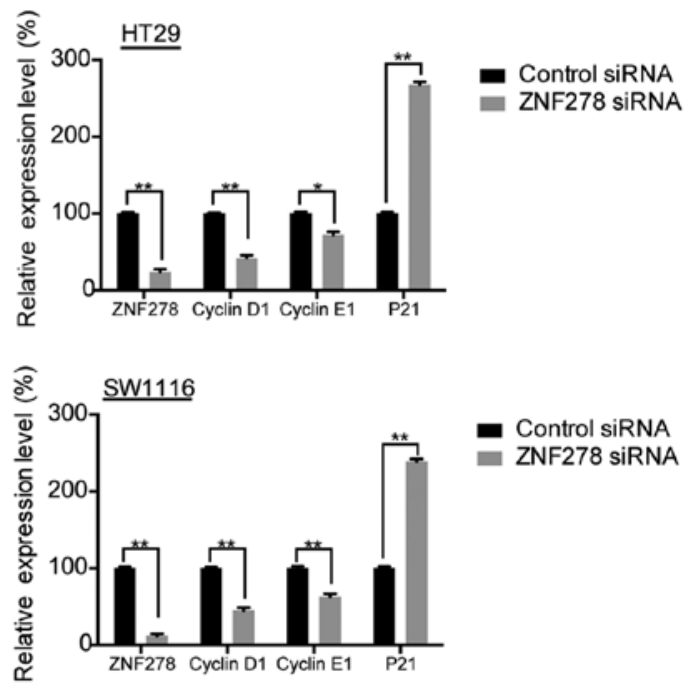

$\mathrm{F}$
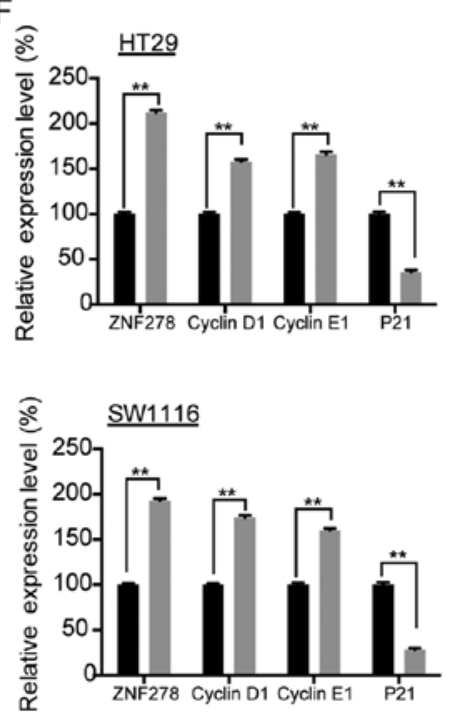

- Control plasmid

ZNF278 plasmid
- Control plasmid

ZNF278 plasmid

Figure 1. Effect of ZNF278 siRNA and plasmid transfection on the cell cycle in CRC cells. (A and B) Real-time PCR was performed to detect the expression of all four ZNF278 variants after ZNF278-siRNA transfection in HT29 and SW1116 cells. (C and D) Western blotting was performed to detect the expression of cell cycle-related proteins and ZNF278 after ZNF278-siRNA transfection in HT29 and SW1116 cells. (E and F) Western blotting was performed to detect the expression of cell cycle-related proteins and ZNF278 after ZNF278 overexpression in HT29 and SW1116 cells. All experiments were repeated at least three times. Data are expressed as the mean $\pm \mathrm{SEM} ;{ }^{*} \mathrm{P}<0.05,{ }^{* *} \mathrm{P}<0.01$.

aforementioned data indicated that ZNF278 was involved in the regulation of $\mathrm{CRC}$ cell cycle progression.
ZNF278 siRNA decreases the proliferation of CRC cells via inhibition of the ERK/MAPK pathway. We 

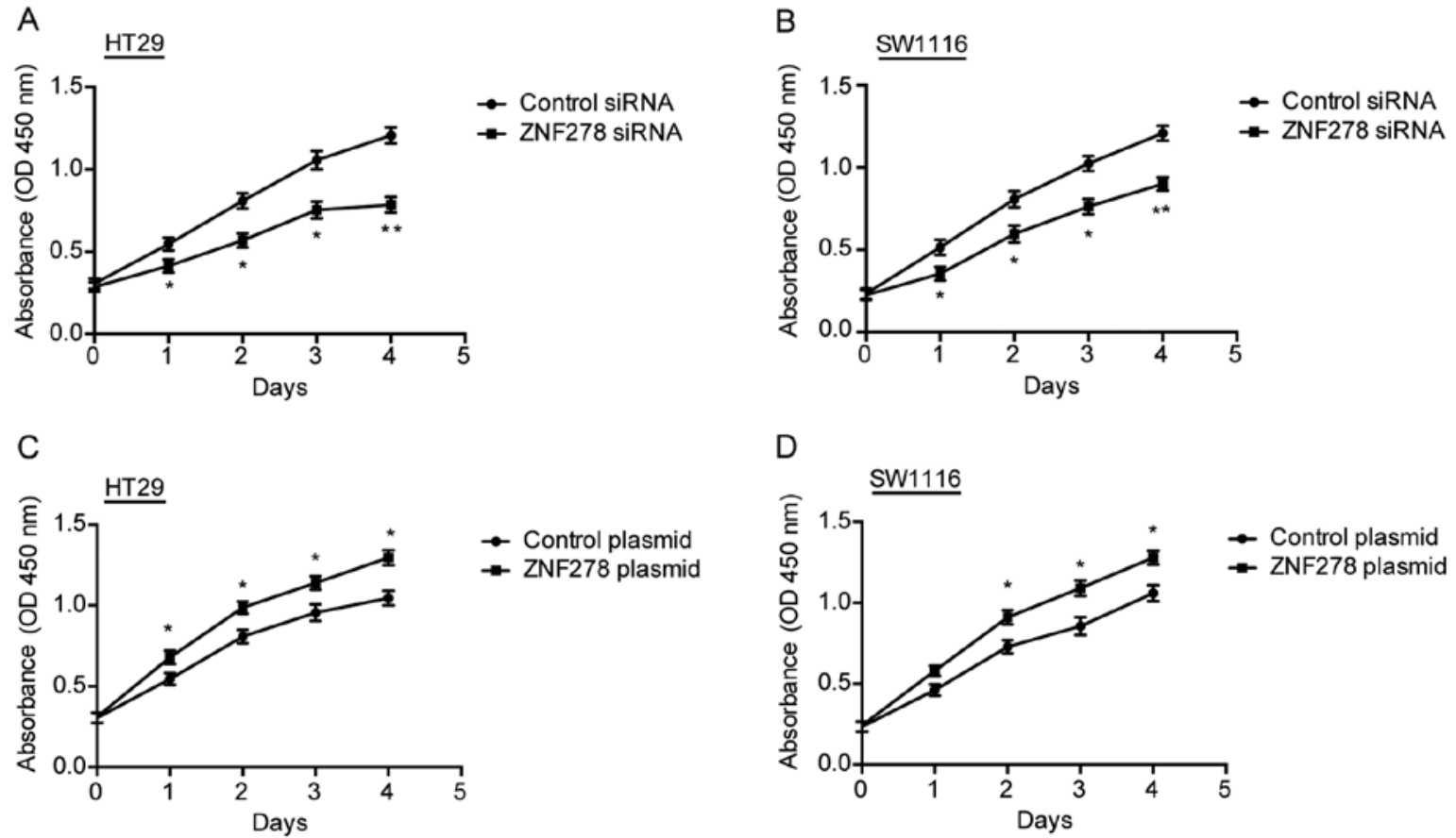

Figure 2. The proliferation rates of CRC cells are significantly different after ZNF278 siRNA or ZNF278 plasmid transfection. (A and B) The proliferation rate was detected after knockdown of ZNF278 in (A) HT29 and (B) SW1116 cells. (C and D) The proliferation rate was detected after ectopic overexpression of ZNF278 in (C) HT29 and (D) SW1116 cells. All experiments were repeated at least three times. Data are expressed as the mean \pm SEM; ${ }^{*} \mathrm{P}<0.05,{ }^{* *} \mathrm{P}<0.01$.

B

A
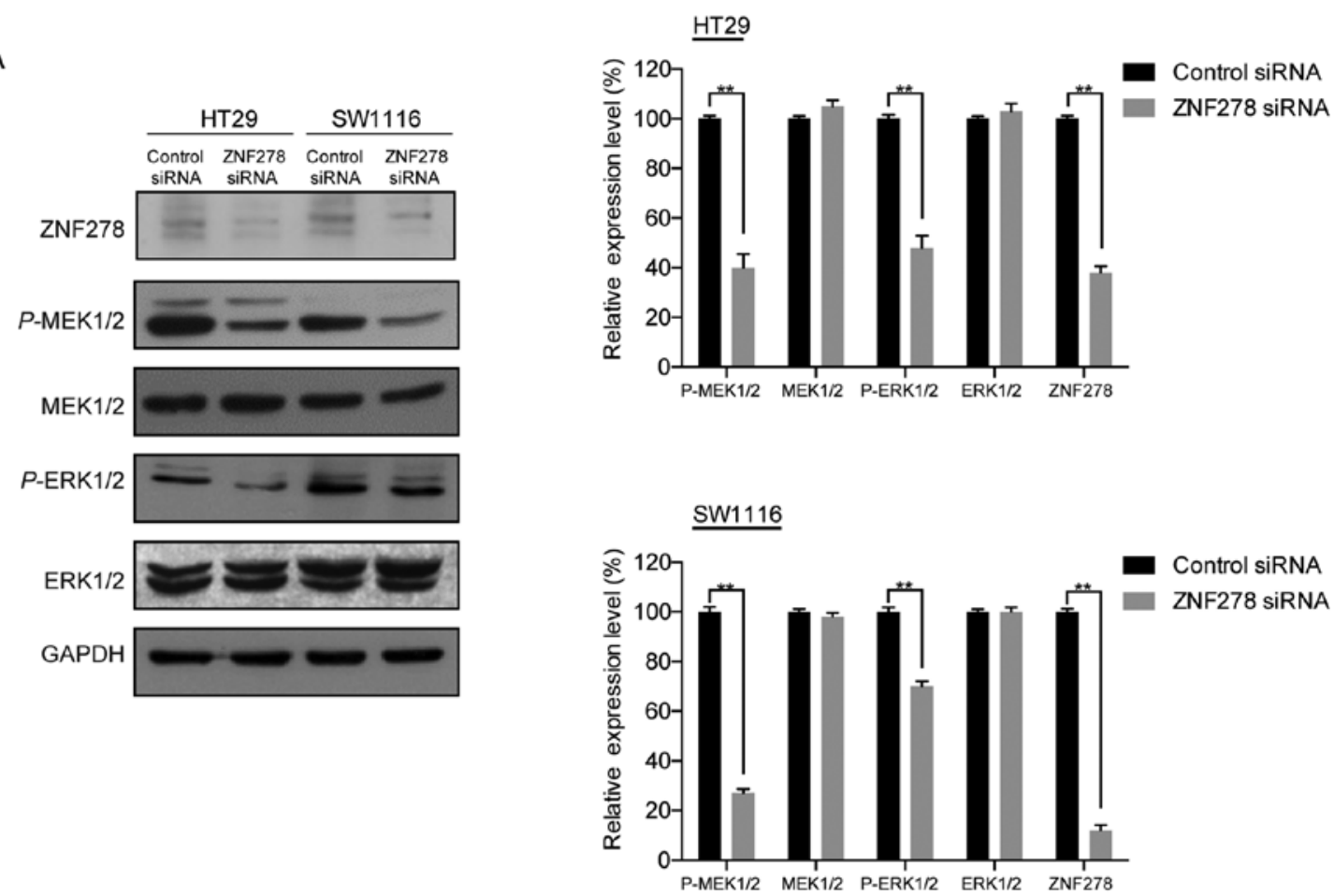

Figure 3. Knockdown of ZNF278 significantly decreased the expression of p-ERK1/2 and p-MEK in CRC cells. (A) Western blotting was performed after ZNF278-siRNA transfection in HT29 and SW1116 cells. (B) The intensity of the western blot bands was quantified by scanning densitometry. All experiments were repeated at least three times. Data are expressed as the mean $\pm \mathrm{SEM} ;{ }^{*} \mathrm{P}<0.05,{ }^{* *} \mathrm{P}<0.01$.

previously demonstrated that transfection of the SW1116 cells with pcDNA3.1-ZNF278 promoted cell growth, and ZNF278-siRNA transfection resulted in a significant inhibition of cell growth (7). In the present study, we further ensured this result in HT29 and SW1116 CRC cells using the CCK-8 assay. Knockdown of ZNF278 significantly inhibited cell proliferation compared to the control siRNA-transfected cells in both HT29 (Fig. 2A) and SW1116 (Fig. 2B) 
A

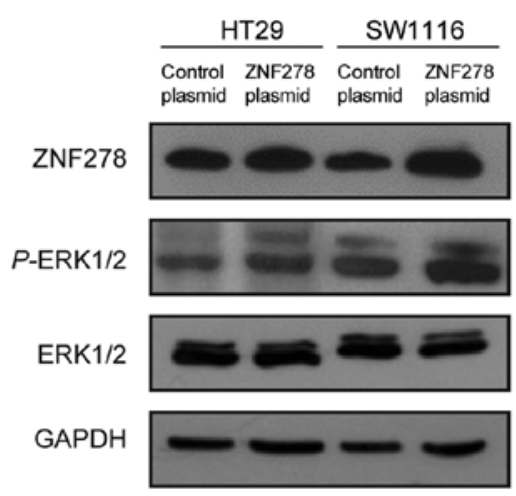

B
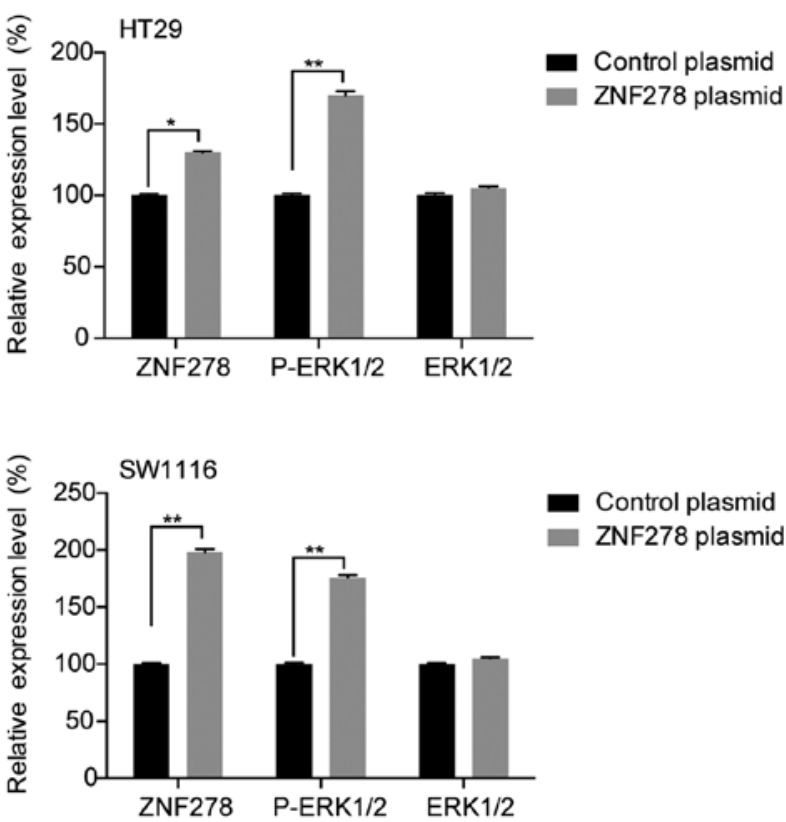

Control plasmid

ZNF278 plasmid

Figure 4. Overexpression of ZNF278 significantly increases the expression of p-ERK1/2 in CRC cells. (A) Western blotting was performed after ZNF278 overexpression in HT29 and SW1116 cells. (B) The intensity of the western blotting bands was quantified by scanning densitometry. All experiments were repeated at least three times. Data are expressed as the mean $\pm \mathrm{SEM} ;{ }^{*} \mathrm{P}<0.05,{ }^{* *} \mathrm{P}<0.01$.

A

$\underline{\mathrm{HT} 29} \rightarrow$ Control plasimd+control siRNA

$\rightarrow$ ZNF278 plasmid+control siRNA

- Control plasimd+ERK2 siRNA

$\rightarrow$ ZNF278 plasmid + ERK2 siRNA

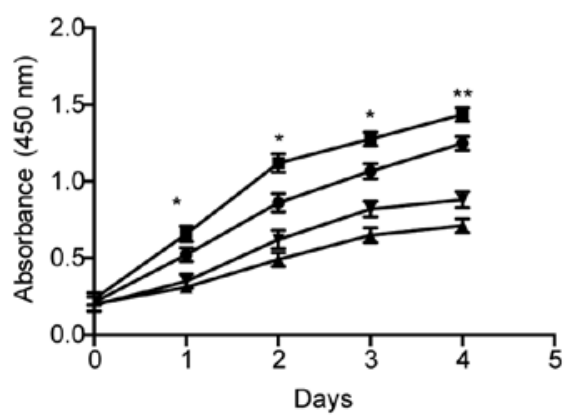

B sw1116 $\rightarrow$ control plasimd+control siRNA

- ZNF278 plasmid+control siRNA

- Control plasimd+ERK2 siRNA

$\rightarrow$ ZNF278 plasmid + ERK2 siRNA

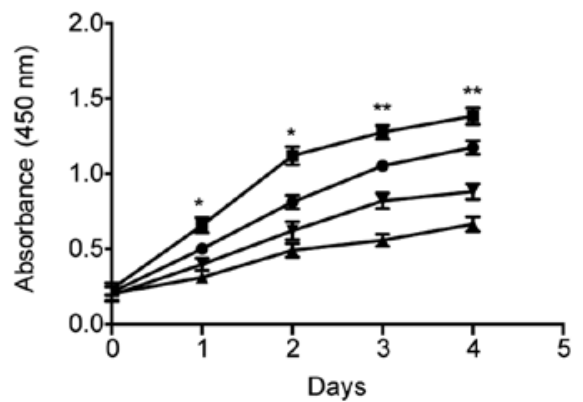

Figure 5. The proliferation rate was detected after ZNF278 overexpression and transfection of ERK2 siRNA in (A) HT29 and (B) SW1116 cells. All experiments were repeated at least three times. Data are expressed as the mean $\pm \mathrm{SEM} ;{ }^{*} \mathrm{P}<0.05,{ }^{* *} \mathrm{P}<0.01$.

cells. Conversely, ectopic overexpression of ZNF278 accelerated cell proliferation in HT29 (Fig. 2C) and SW1116 cells (Fig. 2D). To elucidate the molecular mechanisms by which ZNF278-modulated CRC cell proliferation, we investigated the effects of knockdown of ZNF278 on the extracellular signal-regulated kinase/mitogen-activated protein kinase (ERK/MAPK) pathway, which is frequently aberrantly activated in human cancers and contributes to cell proliferation and survival $(11,12)$. Western blotting revealed that transfection with ZNF278 siRNA significantly decreased ERK1/2 and MEK1/2 phosphorylation in both HT29 and SW1116 cells. However, no detectable changes in the total levels of ERK1/2 and MEK1/2 were observed (Fig. 3A and B). In contrast, ectopic overexpression of ZNF278 induced activation of ERK1/2 and promoted cell proliferation in HT29 and SW1116 cells (Fig. 4A and B). Furthermore, ZNF278 overexpression-induced CRC cell proliferation was markedly blocked by ERK2-siRNA transfection (Fig. 5). Thus, these data suggested that the ERK/MAPK pathway may participate in ZNF278-induced cell proliferation in CRC cells.

The ERK/MAPK pathway may not regulate the expression of ZNF278. We demonstrated that downregulation of ZNF278 inhibited the ERK/MAPK pathway. To investigate whether reciprocal regulation of ZNF278 by the ERK-MAPK pathway occurs, we assessed the expression of ZNF278 in HT29 and SW1116 cells treated with PD98059 (a MEK1 inhibitor) or ERK2 siRNA using western blotting $(13,14)$. Neither PD98059 nor ERK2 siRNA significantly affected the expression of ZNF278 in either HT29 or SW1116 cells (Fig. 6), which indicated that the ERK/MAPK signaling pathway may not participate in the regulation of ZNF278 expression. 
B
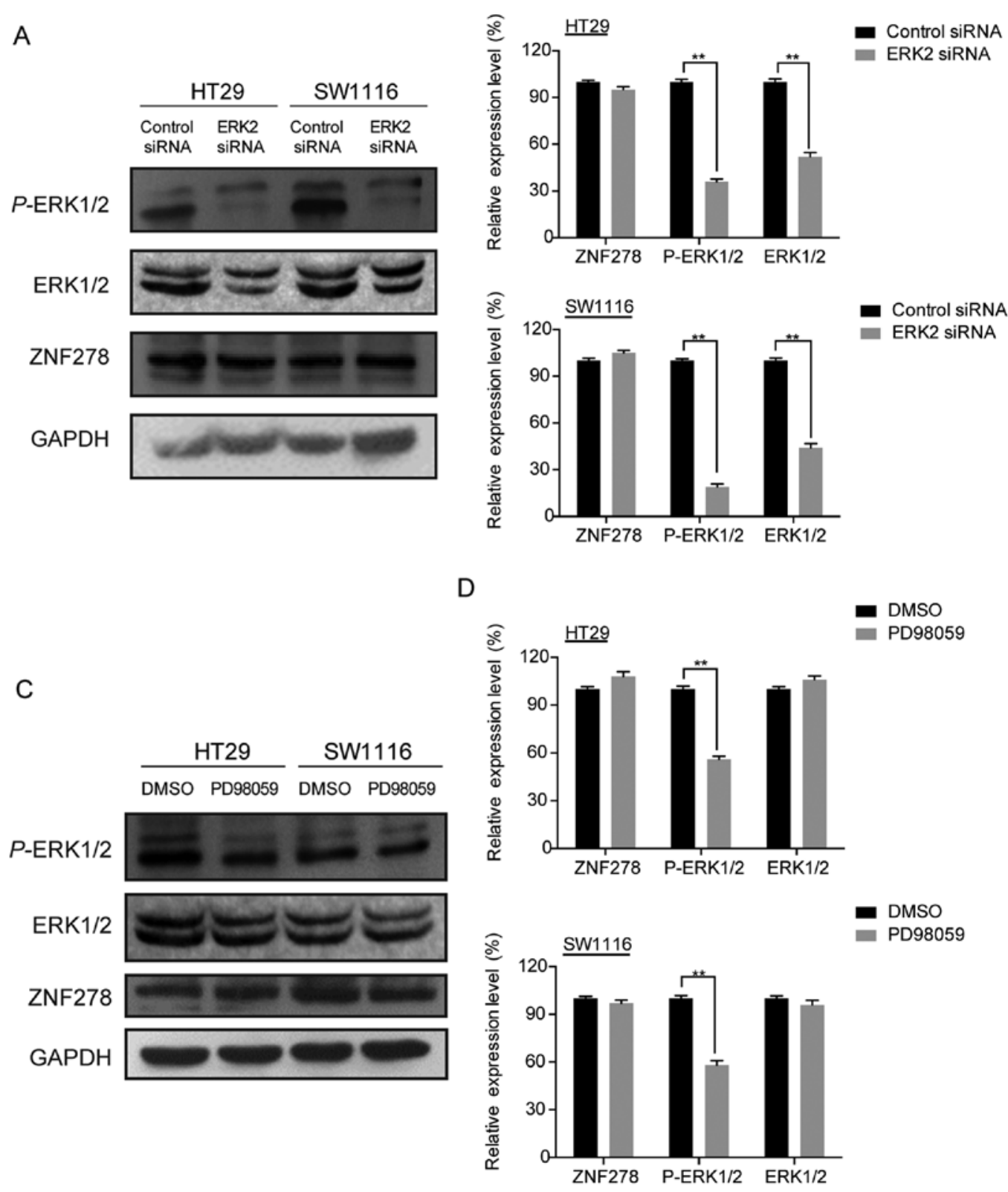

Figure 6. Neither PD98059 nor ERK2 siRNA affect the expression of ZNF278 in CRC cells. (A and B) Western blotting was performed after ERK2-siRNA transfection in HT29 and SW1116 cells. (C and D) Western blotting was performed after PD98059 intervention in HT29 and SW1116 cells. The intensity of the western blot bands was quantified by scanning densitometry. All experiments were repeated at least three times. Data are expressed as the mean \pm SEM; ${ }^{*} \mathrm{P}<0.05,{ }^{* *} \mathrm{P}<0.01$.

\section{Discussion}

Colorectal cancer (CRC) is a common malignant tumor worldwide, with the incidence increasing in Asian countries (15) Aberrant gene expression is involved in colorectal carcinogenesis $(16,17)$. The ZNF278 protein contains an AT-hook DNA-binding motif that usually binds to other DNA-binding structures to play an important role in chromatin modeling and transcription regulation. Its $\mathrm{Poz}$ domain is thought to function as a site for protein-protein interaction and is required for transcriptional suppression $(4,5)$. ZNF278 belongs to the $\mathrm{C} 2 \mathrm{H} 2$-type zinc finger protein family. Various studies have supported that $\mathrm{C} 2 \mathrm{H} 2$-type zinc finger proteins regulate cell proliferation, growth, differentiation, and carcinogenesis (18-21). The ZNF278 protein has typical features of a transcription factor. Recently, a study indicated a critical role of ZNF278 in the control of cell growth and embryonic development (22). Based on previous research, ZNF278 was considered to be an important factor in the physiological state. It has been confirmed that aberrant expression of ZNF278 was related to the development of some diseases, but its cancer-related function as an oncogene or tumor-suppressor remains unclear. In has been reported that, the rearrangement of the ZNF278 gene was detected in small round cell sarcoma (6). Moreover,ZNF278 could be a molecular biomarker and potential target for therapeutic strategies in human testicular germ cell tumors (23). Another study revealed that ZNF278 could be a potential tumor-suppressor in thyroid 
cancer progression (24). We speculated that ZNF278 plays a role as an oncogene or a tumor-suppressor depending on the different molecules interacting with ZNF278 in different tissue cells. ZNF278 may enhance apoptosis or cell survival depending on the different cellular context.

In the previous study, we investigated the possible roles of ZNF278 in colon carcinogenesis (7). We examined the ZNF278 expression level in CRC tissues and corresponding non-cancerous tissues, and found that the ZNF278 expression was significantly higher in cancer tissues compared to the non-cancerous tissues. This revealed that the upregulation of ZNF278 expression may contribute to colorectal tumor carcinogenesis. In order to identify the function of ZNF278, we constructed a wild-type ZNF278 expression vector and transfected to the CRC cell line SW1116. We also performed transient transfection in SW1116 cells with ZNF278 siRNA. In addition, we studied the effects of ZNF278 on the biological functions of $\mathrm{CRC}$ cells with regard to the overexpression and knockdown of ZNF278. The data revealed that ZNF278 increased CRC cell proliferation, and that the knockdown of ZNF278 suppressed cell proliferation and arrested the cell cycle (7). Some previous studies also focused on the effect of ZNF278 knockdown on cell cycle and apoptosis. Ow et al reported that ZNF278 knockdown largely decreased upregulation of apoptotic genes and downregulation of the cell cycle and cellular metabolism genes in embryonic stem cells (ESCs) (25). Tritz et al found that human glioma cells increased their sensitivity to apoptotic stimuli in response to ZNF278 treatment (26). In particular, ZNF278 is a type of zinc finger protein and contains domains involved in DNA-binding and protein-protein interactions (27). It is possible that ZNF278 is involved in some important signaling pathways or regulates the transcription of other important genes. In addition, recent studies revealed that ZNF278 may play biological functions by suppressing the p53 pathway (28-30). It is still unclear whether ZNF278 is involved in other signaling pathways.

In the present study, we investigated the molecular mechanisms by which downregulation of ZNF278 arrested the cell cycle and decreased proliferation of CRC cells. It has been demonstrated that the ERK/MAPK pathway is one of the most important signal transduction pathways, and several key growth factors and proto-oncogenes promote tumor growth by activating this signaling cascade (31-35). Therefore, we examined the effects of ZNF278 on the ERK/MAPK pathway. Knockdown of ZNF278 significantly decreased ERK1/2 and MEK1/2 phosphorylation in HT29 and SW1116 cells; however, no detectable changes in total ERK1/2 and MEK1/2 protein expression were observed. In contrast, ectopic overexpression of ZNF278 increased the phosphorylation of ERK1/2 and promoted HT29 and SW1116 cell proliferation. Moreover, ERK2 siRNA significantly abolished ZNF278 overexpression-induced HT29 and SW1116 cell proliferation. However, the direct link between ZNF278 and the ERK/MAPK pathway warrants further investigation in a future study.

In summary, we found that knockdown of ZNF278 could arrest the CRC cell cycle by decreasing the expression levels of cyclin D1 and E1 and increasing in the expression level of p21. Furthermore, we demonstrated that knockdown of ZNF278 decreased the cell proliferation of CRC cells via inhibition of the ERK/MAPK pathway. Therefore, ZNF278 may serve as a diagnostic molecular marker or potential therapeutic target for CRC treatment.

\section{Acknowledgements}

The present study was supported by grants from the National Natural Science Foundation (nos. 81421001, 81530072 and $81320108024)$ to J.-Y.F., and the National Natural Science Foundation (no. 81001070) to D.-F.S.

\section{References}

1. Jemal A, Bray F, Center MM, Ferlay J, Ward E and Forman D: Global cancer statistics. CA Cancer J Clin 61: 69-90, 2011.

2. Center MM, Jemal A and Ward E: International trends in colorectal cancer incidence rates. Cancer Epidemiol Biomarkers Prev 18: 1688-1694, 2009.

3. Center MM, Jemal A, Smith RA and Ward E: Worldwide variations in colorectal cancer. CA Cancer J Clin 59: 366-378, 2009.

4. Pero R, Lembo F, Palmieri EA, Vitiello C, Fedele M, Fusco A, Bruni CB and Chiariotti L: PATZ attenuates the RNF4-mediated enhancement of androgen receptor-dependent transcription. J Biol Chem 277: 3280-3285, 2002.

5. Fedele M, Benvenuto G, Pero R, Majello B, Battista S, Lembo F, Vollono E, Day PM, Santoro M, Lania L, et al: A novel member of the BTB/POZ family, PATZ, associates with the RNF4 RING finger protein and acts as a transcriptional repressor. J Biol Chem 275: 7894-7901, 2000.

6. Mastrangelo T, Modena P, Tornielli S, Bullrich F, Testi MA, Mezzelani A, Radice P, Azzarelli A, Pilotti S, Croce CM, et al: A novel zinc finger gene is fused to $E W S$ in small round cell tumor. Oncogene 19: 3799-3804, 2000.

7. Tian X, Sun D, Zhang Y, Zhao S, Xiong H and Fang J: Zinc finger protein 278 , a potential oncogene in human colorectal cancer. Acta Biochim Biophys Sin 40: 289-296, 2008.

8. Lu R, Wang X, Chen ZF, Sun DF, Tian XQ and Fang JY: Inhibition of the extracellular signal-regulated kinase/mitogen-activated protein kinase pathway decreases DNA methylation in colon cancer cells. J Biol Chem 282: 12249-12259, 2007.

9. Ye X, Nalepa G, Welcker M, Kessler BM, Spooner E, Qin J, Elledge SJ, Clurman BE and Harper JW: Recognition of phosphodegron motifs in human cyclin $\mathrm{E}$ by the $\mathrm{SCF}^{\mathrm{Fbw}}$ ubiquitin ligase. J Biol Chem 279: 50110-50119, 2004.

10. Sherr CJ: Cancer cell cycles. Science 274: 1672-1677, 1996.

11. Roberts PJ and Der CJ: Targeting the Raf-MEK-ERK mitogenactivated protein kinase cascade for the treatment of cancer. Oncogene 26: 3291-3310, 2007.

12. Hong SK, Yoon S, Moelling C, Arthan D and Park JI: Noncatalytic function of ERK1/2 can promote Raf/MEK/ERK-mediated growth arrest signaling. J Biol Chem 284: 33006-33018, 2009.

13. Crews CM, Alessandrini A and Erikson RL: The primary structure of MEK, a protein kinase that phosphorylates the ERK gene product. Science 258: 478-480, 1992.

14. Cowley S, Paterson H, Kemp P and Marshall CJ: Activation of MAP kinase kinase is necessary and sufficient for PC12 differentiation and for transformation of NIH 3T3 cells. Cell 77: 841-852, 1994.

15. Sung JJ, Lau JY, Goh KL and Leung WK; Asia Pacific Working Group on Colorectal Cancer: Increasing incidence of colorectal cancer in Asia: Implications for screening. Lancet Oncol 6: 871-876, 2005.

16. Fodde R: The $A P C$ gene in colorectal cancer. Eur J Cancer 38: 867-871, 2002.

17. Rochlitz CF, Herrmann R and de Kant E: Overexpression and amplification of c-myc during progression of human colorectal cancer. Oncology 53: 448-454, 1996.

18. Abdollahi A, Pisarcik D, Roberts D, Weinstein J, Cairns P and Hamilton TC: LOT1 (PLAGL1/ZAC1), the candidate tumor suppressor gene at chromosome $6 \mathrm{q} 24-25$, is epigenetically regulated in cancer. J Biol Chem 278: 6041-6049, 2003.

19. Pourquié O: Developmental biology. A macho way to make muscles. Nature 409: 679-680, 2001.

20. Purandare SM, Ware SM, Kwan KM, Gebbia M, Bassi MT, Deng JM, Vogel H, Behringer RR, Belmont JW and Casey B: A complex syndrome of left-right axis, central nervous system and axial skeleton defects in Zic3 mutant mice. Development 129: 2293-2302, 2002. 
21. Yang JJ: A novel zinc finger protein, ZZaPK, interacts with ZAK and stimulates the ZAK-expressing cells re-entering the cell cycle. Biochem Biophys Res Commun 301: 71-77, 2003.

22. Valentino T, Palmieri D, Vitiello M, Simeone A, Palma G, Arra C, Chieffi P, Chiariotti L, Fusco A and Fedele M: Embryonic defects and growth alteration in mice with homozygous disruption of the Patz1 gene. J Cell Physiol 228: 646-653, 2013.

23. Chieffi $P$ and Chieffi S: Molecular biomarkers as potential targets for therapeutic strategies in human testicular germ cell tumors: An overview. J Cell Physiol 228: 1641-1646, 2013.

24. Chiappetta G, Valentino T, Vitiello M, Pasquinelli R, Monaco M, Palma G, Sepe R, Luciano A, Pallante P, Palmieri D, et al: PATZ1 acts as a tumor suppressor in thyroid cancer via targeting p53-dependent genes involved in EMT and cell migration. Oncotarget 6: 5310-5323, 2015.

25. Ow JR, Ma H, Jean A, Goh Z, Lee YH, Chong YM, Soong R, Fu XY, Yang H and Wu Q: Patz1 regulates embryonic stem cell identity. Stem Cells Dev 23: 1062-1073, 2014.

26. Tritz R, Mueller BM, Hickey MJ, Lin AH, Gomez GG, Hadwiger P, Sah DW, Muldoon L, Neuwelt EA and Kruse CA: siRNA Down-regulation of the PATZ1 gene in human glioma cells increases their sensitivity to apoptotic stimuli. Cancer Ther 6: 865-876, 2008.

27. Pero R, Palmieri D, Angrisano T, Valentino T, Federico A, Franco R, Lembo F, Klein-Szanto AJ, Del Vecchio L, Montanaro D, et al: POZ-, AT-hook-, and zinc finger-containing protein (PATZ) interacts with human oncogene B cell lymphoma 6 (BCL6) and is required for its negative autoregulation. J Biol Chem 287: 18308-18317, 2012.

28. Keskin N, Deniz E, Eryilmaz J, Un M, Batur T, Ersahin T, Cetin Atalay R, Sakaguchi S, Ellmeier W and Erman B: PATZ1 is a DNA damage-responsive transcription factor that inhibits p53 function. Mol Cell Biol 35: 1741-1753, 2015.
29. Valentino T, Palmieri D, Vitiello M, Pierantoni GM, Fusco A and Fedele M: PATZ1 interacts with p53 and regulates expression of p53-target genes enhancing apoptosis or cell survival based on the cellular context. Cell Death Dis 4: e963, 2013.

30. Cho JH, Kim MJ, Kim KJ and Kim JR: POZ/BTB and AT-hookcontaining zinc finger protein 1 (PATZ1) inhibits endothelial cell senescence through a p53 dependent pathway. Cell Death Differ 19: 703-712, 2012.

31. Yan L, Gu H, Li J, Xu M, Liu T, Shen Y, Chen B and Zhang G: RKIP and 14-3-3e exert an opposite effect on human gastric cancer cells SGC7901 by regulating the ERK/MAPK pathway differently. Dig Dis Sci 58: 389-396, 2013.

32. De Luca A, Maiello MR, D'Alessio A, Pergameno $M$ and Normanno N: The RAS/RAF/MEK/ERK and the PI3K/AKT signalling pathways: Role in cancer pathogenesis and implications for therapeutic approaches. Expert Opin Ther Targets 16 (Suppl 2): S17-S27, 2012.

33. Levidou G, Saetta AA, Gigelou F, Karlou M, Papanastasiou P, Stamatelli A, Kavantzas N, Michalopoulos NV, Agrogiannis G, Patsouris E, et al: ERK/pERK expression and B-raf mutations in colon adenocarcinomas: Correlation with clinicopathological characteristics. World J Surg Oncol 10: 47, 2012.

34. Santarpia L, Lippman SM and El-Naggar AK: Targeting the MAPK-RAS-RAF signaling pathway in cancer therapy. Expert Opin Ther Targets 16: 103-119, 2012.

35. Fang JY and Richardson BC: The MAPK signalling pathways and colorectal cancer. Lancet Oncol 6: 322-327, 2005. 